\title{
Strong Coupling of the Cyclotron Motion of Surface Electrons on Liquid Helium to a Microwave Cavity
}

\author{
L. V. Abdurakhimov, ${ }^{*}$ R. Yamashiro, A. O. Badrutdinov, and D. Konstantinov \\ Okinawa Institute of Science and Technology (OIST) Graduate University, Onna, Okinawa 904-0495, Japan
}

(Received 22 March 2016; published 27 July 2016)

\begin{abstract}
The strong coupling regime is observed in a system of two-dimensional electrons whose cyclotron motion is coupled to an electromagnetic mode in a Fabry-Perot cavity resonator. Rabi splitting of eigenfrequencies of the coupled motion is observed both in the cavity reflection spectrum and ac current of the electrons, the latter probed by measuring their bolometric photoresponse. Despite the fact that similar observations of Rabi splitting in many-particle systems have been described as a quantum-mechanical effect, we show that the observed splitting can be explained completely by a model based on classical electrodynamics.
\end{abstract}

DOI: 10.1103/PhysRevLett.117.056803

Introduction.-Recent years have been marked by significant interest in the strong coupling of a collection of quantum particles to the electromagnetic modes of a resonator. Besides the traditional systems used in cavity QED experiments such as Rydberg atoms [1-3], the strong coupling regime has been recently studied in various paramagnetic [4-8] and ferromagnetic [9-11] electron spin ensembles, a coupled nuclear-electron spin system [12], as well as two-dimensional electron systems (2DESs) in semiconductors [13-16] and graphene [17,18]. The hallmark of the strong coupling regime is the splitting in the resonator spectrum revealed in the signal reflected from or transmitted through the resonator. In the case of a collection of $N$ quantum particles this splitting scales as $\sqrt{N}$ and is referred to as Rabi splitting [4,19].

Besides a general interest in the fundamental problem of light-matter interaction, a particular interest in the strong coupling regime comes from quantum information processing as strong coupling to a high- $Q$ resonator enables coherent information transfer between, for example, a qubit and spin system excitations [20]. Therefore, most of the recent observations of strong coupling have been interpreted as pure quantum phenomena. However, it is rarely mentioned that strong coupling between a large $N$-particle ensemble and the coherent state of an electromagnetic mode in a resonator can be described completely classically in many cases $[2,15,21]$. Indeed, one needs to introduce nonlinearity to a strongly coupled quantum system in order to create pure quantum states (e.g., a superconducting qubit can be used for the creation of nonclassical states [22]). Otherwise, in a linear system, such as a coupled system of $\mathrm{N}$-particle ensembles and an electromagnetic cavity mode, the problem is equivalent to two coupled harmonic oscillators that exhibit normal-mode splitting when the eigenfrequencies of the uncoupled oscillators coincide [23].

In this Letter, we report the observation of strong coupling between the cyclotron mode of $2 \mathrm{D}$ electrons on the surface of liquid helium and a 3D microwave cavity resonator. The splitting in the eigenspectrum of the coupled motion is observed in the cavity reflection signal, as well as in the ac current of the electrons detected by measuring their bolometric photoresponse. A simple model that uses, on the one hand, an expression for the ac conductivity of the electrons and, on the other hand, the classical equations for the electromagnetic field in the cavity accounts for all of the experimental features including the observed splitting. The square-root scaling of the splitting with the number of electrons follows naturally from our model. Thus, our work reproduces all of the features of the strong coupling regime for a large $N$-particle 2DES but puts it on a completely classical ground.

Experimental setup.-Measurements were performed at a temperature $T \approx 0.2 \mathrm{~K}$ in a dilution refrigerator [Fig. 1(a)]. Liquid helium-4 was condensed into a vacuum-tight cylindrical copper cell with an internal diameter of $40 \mathrm{~mm}$ [Fig. 1(b)]. The cell contained a semiconfocal Fabry-Perot resonator formed by a top spherical mirror and a flat gold-film mirror at the cell bottom. The distance between the two mirrors was $D=7.4 \mathrm{~mm}$. The flat mirror was made in a form of three concentric electrodes (the Corbino disk). Further details can be found elsewhere [24]. The Fabry-Perot resonator was operated in the $\mathrm{TEM}_{003}$ mode, which can be described by a Gaussian beam distribution $[25,26]$. The beam waist $w_{0}$ was calculated to be about $2 \mathrm{~mm}$. The microwave electric field was parallel to the liquid helium surface, and the liquid surface was located in the antinode of the $\mathrm{TEM}_{003}$ mode (at a distance $h \approx 0.85 \mathrm{~mm}$ above the flat mirror) at which the amplitude of the microwave electric field was maximal. The cavity resonance frequency was $\omega_{r} / 2 \pi \approx 88.4 \mathrm{GHz}$, and the $Q$ factor was measured to be about 900 at low temperatures. The resonator was probed by pulse modulated microwave pumping at the frequency $\omega$ with a modulation rate of $5 \mathrm{kHz}$. The microwave power $P_{r}$ reflected from the resonator was detected by an indium antimonide (InSb) hot-electron bolometer and synchronously demodulated by 
(a)

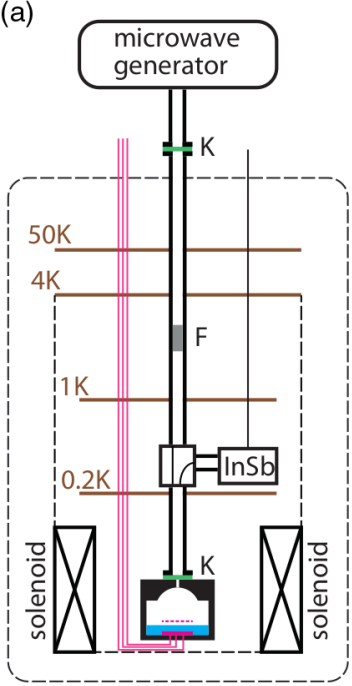

(b)

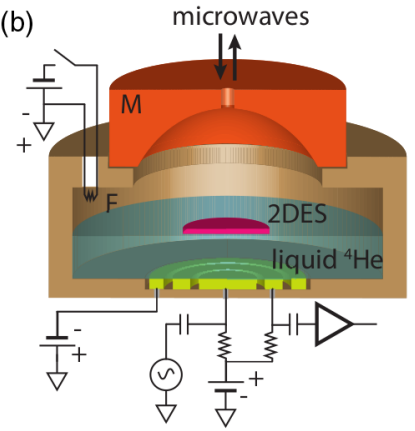

(c)

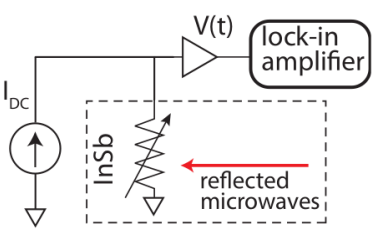

FIG. 1. (a) Schematic diagram of the experimental setup. (b) Sketch of the experimental cell and the Fabry-Perot resonator.

(c) Circuit for the microwave power measurements.

a lock-in amplifier [Fig. 1(c)]. The sensitivity of the InSb bolometer was measured to be about $700 \mathrm{~V} / \mathrm{W}$.

Free electrons were injected into the cell via thermal emission from a tungsten filament mounted inside the cell [see Fig. 1(b)] while a positive voltage $V_{B}$ was applied to the center and middle electrodes of the Corbino disk. In addition to the electrostatic potential created by the biased electrodes, the electrons experience a long-range attractive force towards the free surface of the liquid helium due to its polarizability. On the other hand, the electrons are affected by a short-range repulsive barrier at the liquid surface due to the negative electron affinity of liquid helium caused by the Pauli exclusion principle. Because of the resultant potential well seen by the electrons, a 2DES is formed on the free surface of the liquid helium [27,28]. These surface electrons (SEs) can freely move along the helium surface, but their vertical motion is quantized. The corresponding surface energy levels are described by a hydrogenlike spectrum. The energy difference between the ground surface level and the first excited level is about $0.55 \mathrm{meV}(\approx 6 \mathrm{~K}$ in terms of temperature) in a zero electric pressing field $\left(V_{B}=0\right)$, and it increases with the increase of the pressing field due to the linear Stark effect. Therefore, for the typical temperatures used in the experiment the SEs occupy the ground surface level. The density of the electrons is determined from the condition of the complete screening of the electric field above the surface, $n_{e}=\epsilon_{0} \epsilon V_{B} / e h$, where $\epsilon_{0}$ is the vacuum permittivity, $\epsilon=1.057$ is the liquid helium dielectric constant, and $e$ is the electron charge. The negatively biased outer bottom electrode was used as a guard ring to prevent the electrons from escaping. The magnetic field $B$ perpendicular to the liquid surface was created by a superconducting solenoid, and cyclotron resonance could be excited by the microwave

electrical field when the frequency $\omega$ matched the cyclotron frequency $\omega_{c}=e B / m_{e}$, where $m_{e}$ is the free electron mass.

Strong coupling.-Figure 2(a) shows the reflected microwave power $P_{r}$ obtained with the SEs in the cavity under typical experimental conditions and plotted as a function of the microwave probe frequency $\omega$ and the external magnetic field in units of $\omega_{c}$. A pronounced avoided crossing in the cavity resonance is found near the degeneracy point, that is, the point where the uncoupled cavity mode $\omega_{r}$ would otherwise cross the uncoupled cyclotron-resonance mode $\omega_{c}$. Thus, the observed anticrossing behavior represents the normal-mode splitting in the coupled system of the two oscillators: the cavity mode and the cyclotron motion of the SEs. For the data presented in Fig. 2(a), we find that the value of the splitting between two normal modes at the degeneracy point is about $2 g / 2 \pi=$ $154 \mathrm{MHz}$. The full linewidth of the cavity mode is about $\gamma / 2 \pi=100 \mathrm{MHz}$, and the full linewidth of the cyclotron mode is approximately $\nu / 2 \pi=20 \mathrm{MHz}$. Thus, the cooperativity $C=4 g^{2} / \gamma \nu \approx 12$ is larger than unity, and, hence, the strong coupling regime is realized in our experiment.

The observed avoided crossing is consistent with the Rabi splitting effect, which is typically discussed in the context of similar experiments on strong coupling between quantum particle ensembles and cavity modes [4-14]. In our experiment, the latter would be given by the coupling constant $g$ in the form $g=g_{0} \sqrt{N_{e}}$, where $g_{0}$ is the coupling strength for a single electron, and $N_{e}$ is the total number of electrons coupled to the cavity mode. For nondegenerate SEs occupying the lowest energy level of cyclotron motion the coupling strength is given by $g=\left(e l_{B} E_{0}\right) / \hbar$, where $l_{B}=\sqrt{\hbar / m_{e} \omega_{c}}$ is the magnetic length and $E_{0}$ is the vacuum rms electric field in the cavity. The latter can be estimated as $E_{0}=\sqrt{\hbar \omega / 2 \epsilon_{0} V}$, where $V$ is the cavity mode volume. For comparison with the experiment, the total number $N_{e}$ can be roughly estimated as $N_{e}=n_{e} S$, where $S$ is the characteristic spot size of the microwave Gaussian beam at the liquid helium surface, $S=\pi w_{0}^{2} \approx 12.6 \mathrm{~mm}^{2}$. The mode volume $V_{m}$ for the Gaussian beam, described by the distribution $E(r, z)=E_{0} f(r, z)$, was estimated numerically by integration over the cavity volume as $V_{m}=$ $\int f^{2}(r, z) 2 \pi r d r d z \approx 0.02 \mathrm{~cm}^{3}$. Thus, we obtain the estimation value $g / 2 \pi \approx 200 \mathrm{MHz}$, which is comparable with the experimental value $g / 2 \pi=77 \mathrm{MHz}$. The observed dependence of the splitting on the electron density is consistent with $\sqrt{N_{e}}$ scaling [see the insert in Fig. 3(b)].

In addition to microwave cavity reflection measurements, we also performed a simultaneous detection of the ac current in the 2DES induced by the cavity field. Because it is very difficult to measure such a high frequency current directly, we employed its detection using the electron bolometric photoresponse [29]. The method is based on the effect of the heating induced by the ac current of the SEs on the electron dc resistivity [30]. The latter 

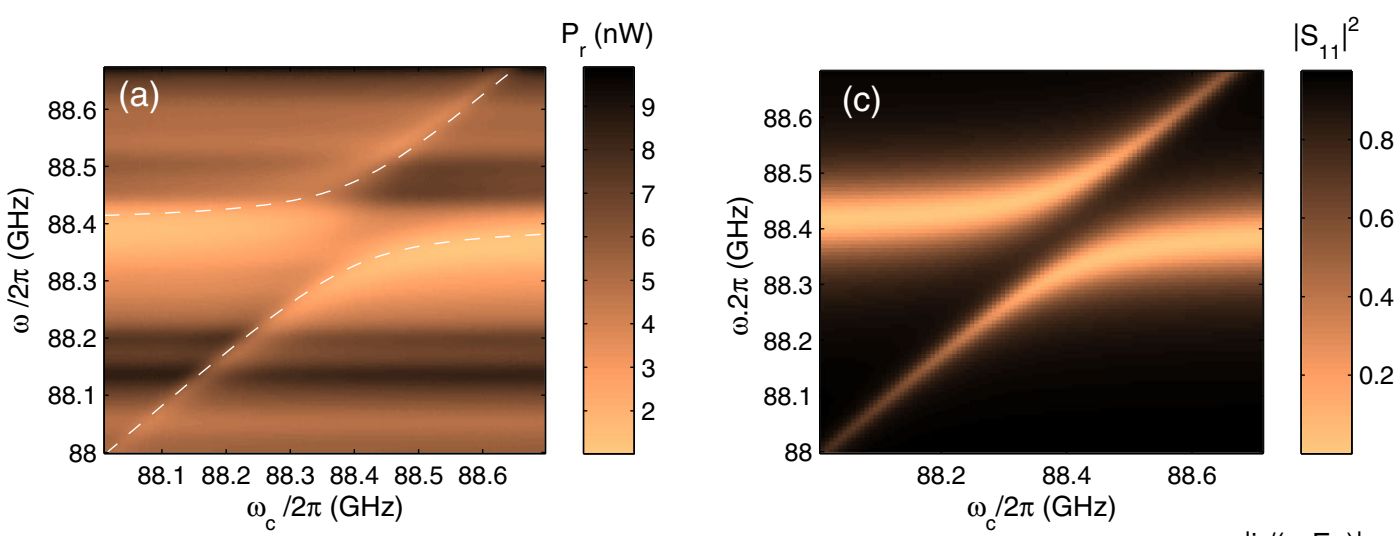

$\operatorname{Re}(\mathrm{Y})(\mathrm{pS})$
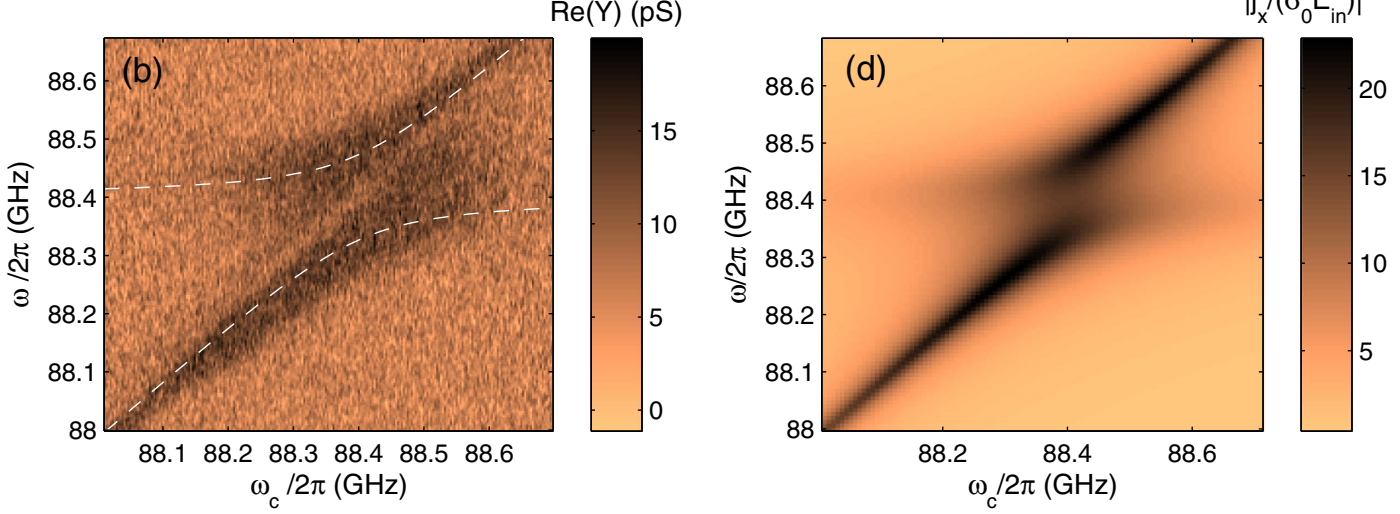

FIG. 2. Left: results of simultaneous measurements of (a) the microwave power reflected from the cavity, $P_{r}$, and (b) the real part of the dc admittance of the cell, $\operatorname{Re}(Y)$, as a function of the microwave probe frequency $\omega$ and the magnetic field in units of $\omega_{c}$. The electron density is $n_{e} \approx 2 \times 10^{8} \mathrm{~cm}^{-2}$, and the microwave probe power is $P_{\text {in }} \approx 180 \mathrm{nW}$. The dashed lines correspond to the calculated eigenfrequencies of the cavity-field-2DES coupled motion with a coupling strength $g / 2 \pi \approx 77 \mathrm{MHz}$. The horizontal stripes in (a) are caused by a parasitic standing wave formed in the waveguide due to a slight impedance mismatching. Right: absolute value of (c) the normalized reflected power $\left|S_{11}\right|^{2}$ and (d) the normalized electron current density $j_{x} /\left(\sigma_{0} E_{\text {in }}\right)$ calculated using the model described in the text for $n_{e}=10^{8} \mathrm{~cm}^{-2}, \gamma=4 \times 10^{8} \mathrm{~s}^{-1}$, and $\nu=10^{8} \mathrm{~s}^{-1}$.

could be probed by measuring the complex admittance of the cell by the capacitive-coupling (Sommer-Tanner) technique $[31,32]$ using the central and middle electrodes of the Corbino disk, see Fig. 1(b). The real part of the admittance, which is proportional to the electron dc resistivity, is plotted in Fig. 2(b) as a function of $\omega$ and $\omega_{c}$. The splitting of the cyclotron resonance mode $\omega=\omega_{c}$, as well as its significant broadening, is clearly observed near the degeneracy point. This measurement directly confirms that the coupling to the microwave cavity mode modifies the electron cyclotron motion and introduces additional damping due to the decay of the cavity field.

Theoretical model.-To understand the above results, we developed a model of the 2DES in a simplified cavity resonator, based on the approach suggested in Ref. [33]. In our model, the motion of the coupled system is described by the following equation [34]

$$
\left(\begin{array}{cc}
\frac{D}{2 c}\left(\omega-\omega_{r}+i \gamma\right) & i \eta_{0} / 2 \\
-\frac{n_{e} e^{2}}{m_{e}}(\nu-i \omega) & (\omega+i \nu)^{2}-\omega_{c}^{2}
\end{array}\right)\left(\begin{array}{c}
E_{x} \\
j_{x}
\end{array}\right)=\left(\begin{array}{c}
E_{\text {in }} \\
0
\end{array}\right),
$$

where $\gamma$ is the total loss rate of the resonator, $D$ is the distance between the resonator mirrors, $c$ is the speed of light, $\eta_{0}$ is the intrinsic impedance of vacuum, $\nu$ is the cyclotron resonance linewidth determined by the electron scattering and Coulomb interaction between the electrons [28,35-37], and $E_{\text {in }} \propto \sqrt{P_{\text {in }}}$ is the electric field component of the microwave probe pump. The above equations relate the microwave electric field $E_{x}$ and electron current density $j_{x}$ in the 2DES plane through the classical electrodynamics relations and electron ac conductivity $\sigma_{x x}=j_{x} / E_{x}$. For $E_{\text {in }}=0$, the solutions of Eq. (1) are two damped eigenmodes with the frequencies plotted in Figs. 2(a) and 2(b) by the dashed lines.

For $E_{\text {in }} \neq 0$, Eq. (1) can be readily solved for a given pump frequency $\omega$. The $S_{11}$ parameter is given by [34]

$$
S_{11}=1+\frac{2\left(\gamma_{\mathrm{ext}}-i \delta \omega_{\mathrm{ext}}\right)}{i\left(\omega-\omega_{r}\right)^{2}-\left(\gamma_{\mathrm{int}}+\gamma_{\mathrm{ext}}\right)-\frac{\sigma_{\mathrm{xx}}}{\varepsilon_{0} D}},
$$

where $\gamma_{\text {int }}$ and $\gamma_{\text {ext }}$ are the internal and external loss rates of the resonator, $\gamma=\gamma_{\mathrm{int}}+\gamma_{\mathrm{ext}}$, and $\delta \omega_{\mathrm{ext}}$ is the resonator 

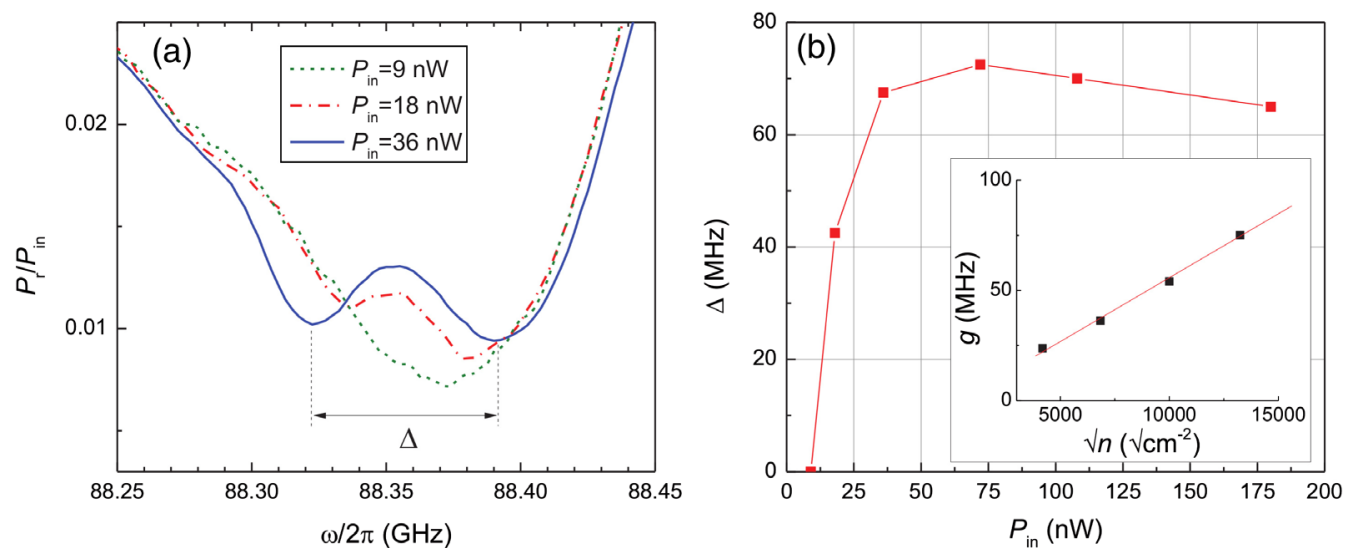

FIG. 3. (a) Normalized spectra of the microwave power reflected from the cavity at several values of the input power. Measurements were performed at the magnetic field corresponding to the pure cyclotron resonance frequency $\omega_{c}=88.35 \mathrm{GHz}$. The electron density is $n_{e} \approx 5 \times 10^{7} \mathrm{~cm}^{-2}$. The splitting appears only at high powers due to the power-induced narrowing of the cyclotron resonance. (b) Power dependence of the splitting $\Delta$ measured as the distance between the two minima of $P_{r}$ near the degeneracy point. Inset: dependence of the coupling strength $g$ on the square root of the electron density, $\sqrt{n_{e}}$, at an input power of $P_{\text {in }} \approx 70 \mathrm{nW}$.

frequency shift due to external coupling. For the sake of comparison with the experiment, $\left|S_{11}\right|^{2}$ and the normalized current density $\left|j_{x} /\left(\sigma_{0} E_{\text {in }}\right)\right|$, where $\sigma_{0}=n_{e} e^{2} /\left(m_{e} \nu\right)$, are shown in Figs. 2(c) and 2(d). Clearly, our model completely accounts for all of the experimental observations.

According to our model the normal-mode splitting at the degeneracy point is given by $2 g=2 \sqrt{n_{e} e^{2} /\left(2 \varepsilon_{0} m_{e} D\right)}$ [34]. Note that this result coincides with the expression for Rabi splitting in terms of the vacuum rms electric field $E_{0}$. Indeed, after multiplying and dividing the above result by $\hbar$, it is straightforward to rewrite it in the form $2 g=$ $\left(2 e l_{B} E_{0}\right) / \hbar$ (see Ref. [34]). We can also represent the coupling constant $g$ in the form $g \propto \sqrt{\alpha n_{e}}$, where $\alpha=$ $e^{2} / c \hbar$ is the fine structure constant. A similar "quantum" representation for the coupling constant (denoted there by $\Omega$ ) was used in Ref. [14] to describe the strong coupling between the cyclotron transition of a 2DES and terahertz resonators. Thus, we demonstrate that, similar to our work, the results of Ref. [14] can be explained by a classical model as well.

A peculiar feature of our experimental results is a strong dependence of the coupling regime on the microwave probe power $P_{\text {in. }}$. The normal-mode splitting becomes noticeable only at a sufficiently high power, as shown on Figs. 3(a) and 3 (b). We suppose that the observed behavior is related to the strong nonmonotonic dependence of the cyclotron resonance linewidth $\nu$ on the microwave power. Indeed, the so called Coulomb narrowing of the cyclotron resonance linewidth with microwave power was observed and explained in terms of electron heating and the increase of the many-electron fluctuating electric field experienced by an electron from its neighbours [36]. This field leads to the suppression of electron scattering within the Landau level, and, therefore, to a reduction in $\nu$. For small powers, the cyclotron resonance linewidth is about $100 \mathrm{MHz}$ [29] and, since $g<\nu, \gamma$, the system is not in the strong coupling regime and normal-mode splitting is not observed. With increasing power, a reduction in $\nu$ allows us to reach the strong coupling regime, and normal-mode splitting is observed. A further increase in power leads to a further increase of the many-electron fluctuating electric field, which eventually assists the inter-Landau-level scattering, resulting in an increase in $\nu[36,37]$. Correspondingly, the observed normal-mode splitting diminishes, as shown in Fig. 3(b). The dependence of the observed splitting on $\nu$ obtained from our model is in good agreement with the experimental observation [34].

Conclusions. - We report the observation of the strong coupling regime between a collection of $2 \mathrm{D}$ electrons on liquid helium and a microwave cavity mode. The reported normal-mode splitting, also referred to as Rabi splitting, is observed in both microwave response and electron transport measurements and shows the correct scaling with the number of particles. We demonstrate that, in contrast with the usual quantum-mechanical description of similar observations in other experiments, our result can be completely explained by a classical model. A similar classical treatment should be able to account for observations of strong coupling in other linear systems. We note that adding a nonlinear quantum system, such as a qubit, to our experiment can provide the possibility to use the pure bosonic system of quantum oscillators on liquid helium for cavity QED experiments and quantum information processing. In addition, the presented experimental method provides the possibility to study intriguing radiation-induced magnetotransport phenomena such as the zero conductance [38] and incompressible states [39] of a 2DES on liquid helium in the regime of strong coupling to a radiation field. 
The work was supported by an internal grant from Okinawa Institute of Science and Technology (OIST) Graduate University. We thank A. Chepelianskii and Y. Kubo for helpful discussions, and V. P. Dvornichenko for technical support.

*1a396@cam.ac.uk

Present address: Microelectronics Group, Cavendish Laboratory, Cambridge University, Cambridge CB3 OHE, United Kingdom.

[1] Y. Kaluzny, P. Goy, M. Gross, J. M. Raimond, and S. Haroche, Phys. Rev. Lett. 51, 1175 (1983).

[2] Y. Zhu, D. J. Gauthier, S. E. Morin, Q. Wu, H. J. Carmichael, and T. W. Mossberg, Phys. Rev. Lett. 64, 2499 (1990).

[3] R. J. Thompson, G. Rempe, and H. J. Kimble, Phys. Rev. Lett. 68, 1132 (1992).

[4] I. Chiorescu, N. Groll, S. Bertaina, T. Mori, and S. Miyashita, Phys. Rev. B 82, 024413 (2010).

[5] D. I. Schuster, A. P. Sears, E. Ginossar, L. DiCarlo, L. Frunzio, J. J. L. Morton, H. Wu, G. A. D. Briggs, B. B. Buckley, D. D. Awschalom, and R. J. Schoelkopf, Phys. Rev. Lett. 105, 140501 (2010).

[6] Y. Kubo, F. R. Ong, P. Bertet, D. Vion, V. Jacques, D. Zheng, A. Dreau, J.-F. Roch, A. Auffeves, F. Jelezko, J. Wrachtrup, M. F. Barthe, P. Bergonzo, and D. Esteve, Phys. Rev. Lett. 105, 140502 (2010).

[7] R. Amsuss, C. Koller, T. Nobauer, S. Putz, S. Rotter, K. Sandner, S. Schneider, M. Schrambock, G. Steinhauser, H. Ritsch, J. Schmiedmayer, and J. Majer, Phys. Rev. Lett. 107, 060502 (2011).

[8] E. Abe, H. Wu, A. Ardavan, and J. J. L. Morton, Appl. Phys. Lett. 98, 251108 (2011).

[9] H. Huebl, C. W. Zollitsch, J. Lotze, F. Hocke, M. Greifenstein, A. Marx, R. Gross, and S. T. B. Goennenwein, Phys. Rev. Lett. 111, 127003 (2013).

[10] Y. Tabuchi, S. Ishino, T. Ishikawa, R. Yamazaki, K. Usami, and Y. Nakamura, Phys. Rev. Lett. 113, 083603 (2014).

[11] X. Zhang, C.-L. Zou, L. Jiang, and H. X. Tang, Phys. Rev. Lett. 113, 156401 (2014).

[12] L. V. Abdurakhimov, Y. M. Bunkov, and D. Konstantinov, Phys. Rev. Lett. 114, 226402 (2015).

[13] D. Hagenmuller, S. De Liberato, and C. Ciuti, Phys. Rev. B 81, 235303 (2010).

[14] G. Scalari, C. Maissen, D. Turčinkovà, D. Hagenmüller, S. De Liberato, C. Ciuti, C. Reichl, D. Schuh, W. Wegscheider, M. Beck, and J. Faist, Science 335, 1323 (2012).

[15] V. M. Muravev, I. V. Andreev, I. V. Kukushkin, S. Schmult, and W. Dietsche, Phys. Rev. B 83, 075309 (2011);
V. M. Muravev, P. A. Gusikhin, I. V. Andreev, and I. V. Kukushkin, Phys. Rev. B 87, 045307 (2013).

[16] Q. Zhang, M. Lou, X. Li, J. Reno, W. Pan, J. Watson, M. Manfra, and J. Kono, arXiv:1604.08297.

[17] D. Hagenmuller and C. Ciuti, Phys. Rev. Lett. 109, 267403 (2012).

[18] L. Chirolli, M. Polini, V. Giovannetti, and A. H. MacDonald, Phys. Rev. Lett. 109, 267404 (2012).

[19] G. S. Agarwal, Phys. Rev. Lett. 53, 1732 (1984).

[20] A. Imamoglu, Phys. Rev. Lett. 102, 083602 (2009).

[21] L. Bai, M. Harder, Y. P. Chen, X. Fan, J. Q. Xiao, and C.-M. Hu, Phys. Rev. Lett. 114, 227201 (2015).

[22] M. Hofheinz, E. M. Weig, M. Ansmann, R. C. Bialczak, E. Lucero, M. Neeley, A. D. O'Connell, H. Wang, J. M. Martinis, and A. N. Cleland, Nature (London) 454, 310 (2008).

[23] S. Haroche and J.-M. Raimond, Exploring the Quantum: Atoms, Cavities and Photons (Oxford University Press, Oxford, 2006), Chap. 3.

[24] R. Yamashiro, L. V. Abdurakhimov, A. O. Badrutdinov, Y. P. Monarkha, and D. Konstantinov, Phys. Rev. Lett. 115, 256802 (2015).

[25] H. Kogelnik and T. Li, Appl. Opt. 5, 1550 (1966).

[26] We use the notation $\mathrm{TEM}_{00 q}$ with $q=0,1,2, \ldots$, where the number of half wavelengths is $q+1$. See the details in Ref. [25].

[27] E. Y. Andrei, Two-Dimensional Electron Systems on Helium and other Cyrogenic Substrates, edited by E. Y. Andrei (Kluwer Academic Publishers, Dordrecht, 1997).

[28] Y. Monarkha and K. Kono, Two-Dimensional Coulomb Liquids and Solids (Springer, Berlin, 2004).

[29] V. S. Edel'man, JETP Lett. 25, 394 (1977).

[30] T. Aoki and M. Saitoh, J. Phys. Soc. Jpn. 48, 1929 (1980).

[31] R. Mehrotra and A. Dahm, J. Low Temp. Phys. 67, 115 (1987).

[32] L. Wilen and R. Giannetta, J. Low Temp. Phys. 72, 353 (1988).

[33] V. Shikin, JETP Lett. 75, 29 (2002).

[34] See Supplemental Material at http://link.aps.org/ supplemental/10.1103/PhysRevLett.117.056803 for the details of our classical model.

[35] M. I. Dykman, C. Fang-Yen, and M. J. Lea, Phys. Rev. B 55, 16249 (1997).

[36] E. Teske, Y. P. Monarkha, M. Seck, and P. Wyder, Phys. Rev. Lett. 82, 2772 (1999).

[37] Y. P. Monarkha, E. Teske, and P. Wyder, Phys. Rev. B 62, 2593 (2000).

[38] D. Konstantinov and K. Kono, Phys. Rev. Lett. 105, 226801 (2010).

[39] A. D. Chepelianskii, M. Watanabe, K. Nasyedkin, K. Kono, and D. Konstantinov, Nat. Commun. 6, 7210 (2015). 